Mayo 2020

\title{
Revisiones sistematizadas en Ciencias Humanas y Sociales. 3: Análisis y Síntesis de la información cualitativa
}

\author{
Lluís Codina \\ Universitat Pompeu Fabra \\ lluis.codina@upf.edu \\ ORCID: 0000-0001-7020-1631
}

DOI: 10.31009/methodos.2020.i01.07

Codina, L. (2020). Revisiones sistematizadas en Ciencias Humanas y Sociales. 3: Análisis y Síntesis de la información cualitativa. En: Lopezosa, C.; Díaz-Noci, J.; Codina, L. (ed.). Anuario de Métodos de Investigación en Comunicación Social, n.1 (p.73-87). Barcelona: DigiDoc-Universitat Pompeu Fabra 



\title{
Revisiones sistematizadas en Ciencias Humanas y Sociales. 3: Análisis y Síntesis de la información cualitativa
}

\author{
Lluís Codina \\ Universitat Pompeu Fabra \\ lluis.codina@upf.edu \\ ORCID: 0000-0001-7020-1631
}

\section{RESUMEN}

El Análisis y la Síntesis son las dos últimas fases de una revisión sistematizada que sigue el Framework SALSA, parte a su vez del (meta)framework ReSiste-CSH que estamos presentando en esta serie de tres capítulos, de la cual este es el último. El Análisis se ocupa de proveer un medio de examen de los documentos que debe ser aplicado a todos por igual, con el objetivo de tener una base de comparación y, sobre todo, de Síntesis. La Síntesis, entonces es la última de las fases en el mencionado Framework SALSA. En un marco más amplio, tendríamos aún una fase posterior de presentación de la revisión o del estado de la cuestión, según el destino concreto de nuestra revisión.

\section{PALABRAS CLAVE}

Revisiones sistemáticas, Revisiones sistematizadas, Revisiones bibliográficas, Framework SALSA, Framework ReSiste-CHS, Análisis de documentos, Síntesis.

\section{Systematized revisions in Human and Social Sciences. Third part: Analysis and Synthesis of Qualitative Information}

\section{ABSTRACT}

The Analysis and the Synthesis are the last two phases of a systematized review that follows the SALSA Framework, part of the ReSiste-CSH (meta) framework that we are presenting in this series of three chapters, of which this is the last one. The Analysis is concerned with providing a means of examining the documents that must be applied to all equally, with the aim of having a base of comparison and, above all, of Sinthesis. The Synthesis, then is the last of the phases in the mentioned SALSA Framework. In a broader framework, we would still have a subsequent phase of presentation of the review or the state/s of the art, according to the specific destination of our review.

\section{KEYWORDS}

Systematic reviews, systematized reviews, literary reviews, SALSA framework, ReSiste-CHS framework, Document analysis, Synthesis.

\section{Revisions sistematitzades en Ciències Humanes i Socials. Tercera part:: Anàlisi i Síntesi de la informació qualitativa}

\begin{abstract}
RESUM
L'Anàlisi i la Síntesi són les dues últimes fases d'una revisió sistematitzada que segueix el Framework SALSA, part al seu torn de l'(meta) framework resisteix-CSH que estem presentant en aquesta sèrie de tres capítols, de la qual aquest és l'últim. L'Anàlisi s'ocupa de proveir un mitjà d'examen dels documents que ha de ser aplicat a tots per igual, amb l'objectiu de tenir una base de comparació i, sobretot, de Síntesi. La Síntesi, llavors és l'última de les fases en l'esmentat Framework SALSA. En un marc més ampli, tindríem encara una fase posterior de presentació de la revisió o de l'estat de la qüestió, segons la destinació concreta de la nostra revisió.
\end{abstract}

\section{PARAULES CLAU}

Revisions sistemàtiques, Revisions sistematitzades, Revisions bibliogràfiques, Framework SALSA, Framework resisteix-CHS, Anàlisi de documents, Síntesi 


\section{Introducción}

En las revisiones sistematizadas, el Análisis y la Síntesis corresponden a las dos últimas fases del framework SALSA, que a su vez forma parte del (meta) framework más amplio, ReSiste-SCH, que estamos presentando en esta serie de tres capítulos.

Ambas fases se aplican sobre el banco de documentos que hemos seleccionado gracias a las fases anteriores de Búsqueda y Evaluación. Para el Análisis necesitamos aplicar alguna clase de procedimiento que nos ayuda a tratar de forma similar cada uno de los documentos, mientras que la fase de Síntesis se ocupa de reunir los principales resultados en un resultado final.

Un aspecto importante a considerar es que las recomendaciones y propuestas en estas dos fases, se apartan de la relativa homogeneidad de las dos anteriores.

Como sabemos, las revisiones sistemáticas se centran en la evaluación de la eficiencia de intervenciones, programas o tratamientos de algún tipo (que afecta a una población o colectivo, como un grupo de edad, o un tipo de pacientes, por ejemplo). Esto les permite homogeneizar considerablemente todas las fases.

En cambio, en las revisiones bibliográficas sistematizadas, no existe ninguna restricción a priori ni sobre los temas ni sobre los objetivos ni sobre el contexto de la misma, ya que de lo contrario no podrían cumplir su misión, que es precisamente dar soporte a una amplia variedad de objetivos.

Las revisiones bibliográficas se consideran uno de los componentes obligados (y principales) de tesis de máster o de doctorado, normalmente, en forma de capítulo (o capítulos) de estados de la cuestión.

Igualmente, son un componente obligado de las memorias para solicitar financiación en proyectos de investigación. Adicionalmente, los evaluadores de artículos científicos esperan que estos que incluyan un apartado con los antecedentes relevantes a su investigación, lo cual es una forma, aunque más ligera, de estado de la cuestión.

Todo lo anterior sería imposible de llevar a cabo si las revisiones bibliográficas no pudieran aplicarse a todo tipo de escenario y objetivos. Pero esta flexibilidad, impide a la vez la homogeneización excesiva de estas dos fases, cosa que sería altamente disfuncional.

Aun así, como veremos a continuación, y gracias a la labor de un amplio grupo de autores que se han ocupado del tema, podremos presentar algunas propuestas de sistematización, de manera que estas dos fases dispongan también de garantías de calidad y solidez en sus resultados.

\section{Fundamentos académicos}

Para esta entrega en concreto nos apoyaremos sobre todo en cuatro aportaciones que han tratado estos temas desde la perspectiva de las investigaciones y los trabajos académicos en general, por tanto, hacen propuestas que denominaremos de amplio espectro, es decir, susceptibles de ser aplicadas, a priori, a cualquier ámbito de investigación dentro de las Ciencias Humanas y Sociales. Se trata de las siguientes:

- Hart, Chris. Doing a Literature Review. London: Sage, 2008.

Como ya indicamos en la primera de las entregas, donde presentamos esta obra por primera vez, se trata de una de las aportaciones sobre el tema más influyente y, consecuentemente, más citada. Aunque este autor no recurre al término "sistemática" o "sistematizada", sus propuestas se ajustan perfectamente a la filosofía de esta clase de revisiones. En este apartado es especialmente útil porque su foco son las revisiones bibliográficas que deben formar parte de trabajos académicos más amplios, enfoque que es también el que adoptamos aquí.

- Bloomberg, L.D.; Volpe, M. "Developing and Presenting Your Literature Review". En: Bloomberg, L.D.; Volpe, M. Completing your Qualitative Dissertation: A Road Map from Beggining to End. London: Sage, 2016, pp. 103-142.

Por su parte, en este caso nos centramos en uno de los capítulos (el 6) de una de las mejores obras sobre como llevar a cabo con éxito una tesis doctoral. En este caso, las autoras utilizan específicamente el término "sistemática" para sus propuestas, pero en el contexto, como decimos, de otra obra mayor, lo que encaja perfectamente con nuestro enfoque.

- Miles, M.B; Huberman, M.A; Saldaña, J. Qualitative Data Analysis: A Method Sourcebook. London: Sage, 2014.

Ciertamente, muchos autores hacen recomendaciones sobre el uso de la visualización en la presentación de resultados, pero pocos como Miles y sus colegas (hasta donde nosotros sabemos) han sistematizado el uso de tales de tales instrumentos para cinco funciones básicas: explorar, describir, ordenar, explicar y predecir. Por este motivo, para nosotros es otra de las obras seminales para estos apartados.

- Noblit, G. W.; Hare, R. D. Meta-Ethnography: Synthetising Qualitative Studies. London: Sage, 1988. 
Esta obra seminal, eminentemente centrada en los estudios etnográficos, los autores pusieron las bases para un procedimiento que después se ha utilizado ampliamente en estudios de tipo cualitativo. Nos referimos a los tres tipos de síntesis que proponen y explican muy bien en su obra: (1) la traducción recíproca, (2) la síntesis de refutación y (2) la línea argumental. La primera consiste en identificar trabajos que comparten los mismos conceptos, aunque los identifiquen con diferentes nombres; la segunda se refiere a identificar trabajos que presentan teorías que refutan a otras, y la línea de argumento corresponde a la idea más intuitiva de diversos trabajos que aportan ideas que se apoyan y complementan en la interpretación o comprensión de un fenómeno.

Una vez presentados los antecedentes anteriores, pasamos a considerar las dos fases que nos quedaban pendientes, el Análisis y la Síntesis.

\section{Análisis}

El objetivo de esta fase es llevar a cabo un análisis sistematizado, por tanto, aplicando el mismo esquema, a cada una de las obras que forman parte del banco de documentos.

Se trata de producir un extracto en forma de documento individual o ficha para cada una de las obras analizadas, pero de forma que también sea posible trasladar toda o parte de la información extraída en forma de tablas e incluso de diagramas.

Los elementos que componen el esquema de análisis deben ajustarse según los objetivos y los temas de la revisión o del trabajo académico más amplio en el que se inscriben.

Para el caso en el que nos centramos en esta serie, a saber, las revisiones bibliográficas destinadas a estados de la cuestión para tesis doctorales, de máster o solicitudes de financiación de proyectos, podemos utilizar un conjunto de elementos que pueden servir para casi cualquier investigación.

En concreto, en el Framework ReSiste-CHS, se propone la ficha o esquema de análisis para cada documento que mostramos a continuación.

\section{Ficha de análisis de documentos. Esquema general}

1 Referencia Referencia completa del documento completa de (Autor, Título, Fuente, etc.)

la fuente

\begin{tabular}{|c|c|c|}
\hline 2 & $\begin{array}{l}\text { Resumen } \\
\text { informativo }\end{array}$ & $\begin{array}{l}\text { Algunos resúmenes de artículos científicos } \\
\text { y otras clases de documentos, pueden } \\
\text { ser indicativos (no entran en los resultados } \\
\text { ni ofrecen elementos de discusión). Este } \\
\text { resumen, en cambio debe ser informativo } \\
\text { incluyendo objeto de estudio, metodo- } \\
\text { logía y principales resultados obtenidos. } \\
\text { Extensión recomendada: } 300 \text { palabras. }\end{array}$ \\
\hline 3 & $\begin{array}{c}\text { Tipo de } \\
\text { investigación/ } \\
\text { Métodos de } \\
\text { obtención de } \\
\text { datos }\end{array}$ & $\begin{array}{l}\text { Indicación del tipo de investigación (cualita- } \\
\text { tiva, cuantitativa, teórica, aplicada, estudio de } \\
\text { caso, etc.) y métodos de obtención de datos. }\end{array}$ \\
\hline 4 & $\begin{array}{l}\text { Principales } \\
\text { aportaciones }\end{array}$ & $\begin{array}{l}\text { Indicación de las principales aportaciones del } \\
\text { trabajo: terminología, modelos, conceptos, } \\
\text { diseño experimental, resultados, etc. }\end{array}$ \\
\hline 5 & $\begin{array}{l}\text { Valor, } \\
\text { originalidad o } \\
\text { aspectos más } \\
\text { relevantes }\end{array}$ & $\begin{array}{l}\text { Identificación de los aspectos que } \\
\text { otorgan mayor valor al trabajo: el } \\
\text { rigor, la escritura, los gráficos, etc. }\end{array}$ \\
\hline 6 & $\begin{array}{l}\text { Comentarios } \\
\text { y síntesis } \\
\text { valorativa }\end{array}$ & $\begin{array}{l}\text { Notas y comentarios adicionales } \\
\text { de tipo valorativo/crítico. }\end{array}$ \\
\hline 7 & $\begin{array}{l}\text { Palabras } \\
\text { clave }\end{array}$ & $\begin{array}{l}\text { Palabras clave que ayuden a } \\
\text { categorizar el trabajo. }\end{array}$ \\
\hline
\end{tabular}

\section{Observaciones}

- Para 1 debe utilizarse la norma de citación que después se aplicará a la presentación de la bibliografía final, con el fin de evitar incoherencias.

- En 2, debe evitarse a toda costa la opción de "copiar y pegar" los resúmenes acompañan a las publicaciones académicas. El resumen debe ser original, marcando las frases literales mediante comillas, y debe atribuir las ideas a los autores correspondientes. Debe ser de tipo informativo (no indicativo), y por tanto debe incluir el objeto de estudio, los objetivos, la metodología y los resultados principales.

- En 3 la idea principal es poder caracterizar el tipo de aportación en términos como los señalados, que van más allá de clasificar los trabajos como cualitativos o cuantitativos. En caso necesario, los campos 2 y 3 pueden fusionarse.

- Por lo que hace a $\mathbf{4}$ y $\mathbf{5}$, a veces puede ser difícil diferenciar entre estos dos factores, por lo que puede ser que tenga sentido unificar estos dos campos en algunos casos.

- El apartado 6 es optativo, tal como señalamos, y consiste en añadir alguna clase de síntesis o valoración crítica, lo que hace que sea distinta del resumen del punto 2 . 
- El apartado 7 puede tener utilidad en algunas investigaciones, porque las palabras clave pueden ayudar a relacionar trabajos con ámbitos sectoriales (radio, televisión, Internet, etc.), geográficos o de cualquier otro tipo según la investigación y el proyecto concreto. En tal caso, será imprescindible contar con una lista de tales palabras clave e, idealmente, de una taxonomía.

\section{Organización en formato de tabla}

Para convertir las fichas individuales anteriores en tablas, podemos usar varias tablas unidas por una columna ID común. Por ejemplo, la primera tabla asigna un número de identificación (ID) a cada referencia del banco de documentos.

\section{Tabla 1}

$$
\text { ID Referencia }
$$

Después, podemos utilizar varias tablas con el ID común y algunos de los demás campos. Por ejemplo, en la tabla siguiente, utilizamos tres de los parámetros de análisis., como el tipo de investigación, las principales aportaciones y las palabras clave:

Tabla 2

\begin{tabular}{|l|c|c|c|}
\hline ID & $\begin{array}{c}\text { Tipo de } \\
\text { investigación }\end{array}$ & $\begin{array}{c}\text { Principales } \\
\text { aportaciones }\end{array}$ & Palabras clave \\
\hline
\end{tabular}

\section{Adaptaciones y ejemplos}

El anterior esquema es, como venimos señalando, de amplio espectro, de modo que se puede aplicar incluso cuando el banco de documentos esté formado por trabajos de características muy heterogéneas.

En otros casos, debe ser posible adaptar el esquema con elementos de análisis más específicos. Por ejemplo, en una revisión reciente sobre trabajos vinculados con el así llamado periodismo computacional, se utilizó este otro esquema:

\section{Esquema para analizar un banco de documentos sobre periodismo computacional}

\begin{tabular}{|l|l|}
\hline 1 & Referencia \\
\hline 2 & Resumen y aportaciones más destacadas \\
3 & Palabras clave \\
\hline 4 & Tipo de trabajo \\
\hline 5 & Sector de la comunicación \\
\hline 6 & Dimensión afectada \\
\hline
\end{tabular}

Fuente: Mari Vàllez, Luís Codina, 2018.

\section{Observaciones:}

- El apartado 2, se utilizó para incluir una síntesis de cada documento compuesta por un resumen y las aportaciones más destacadas.

- El apartado 4 nos permitió retener las características del tipo de trabajo, ya que el banco de documentos estaba compuesto por informes de centros de I+D y por artículos de revistas indexadas.

- El apartado 5 sirvió para indicar el sector de la comunicación afectado: prensa, agencias de noticias, televisión, etc.

- En el 6 permitió anotar cuál (o cuáles) de las dimensiones del periodismo computacional estaban tratadas en el documento: robot periodista, periodismo aumentado, periodismo estructurado, etc.

- Por último, el apartado 7 permitió reseñar países o regiones en el caso que el documento se centrase en algún país o zona geográfica.

\section{Los esquemas de Blomberg y Dale (2016)}

Por su parte, Bloomberg y Dale (2016) proponen dos esquemas distintos, según se trate de analizar artículos que presentan resultados de una investigación o artículos de tipo teórico. Los vemos en las siguientes capturas (Figuras 1 y 2)

La captura precedente (Figura 1) corresponde a artículos basados en investigaciones, mientras que la siguiente muestra una propuesta de esquema para artículos de tipo teórico o conceptual:

\section{Esquema de Emerald Publishing}

Se da la circunstancia de que el importante Grupo editorial Emerald Publishing (unas 600 revistas) utiliza un esquema para articular los resúmenes de sus revistas académicas que consideramos que se puede considerar de utilidad para revisiones sistematizadas.

Los componentes son los siguientes:

\begin{tabular}{|l|l|}
\hline 1 & Propósito \\
\hline 2 & Diseño/Metodología/Aproximación \\
\hline 3 & Resultados \\
\hline 4 & Originalidad/Nalor \\
\hline Fuente: Emerald Publishing
\end{tabular}




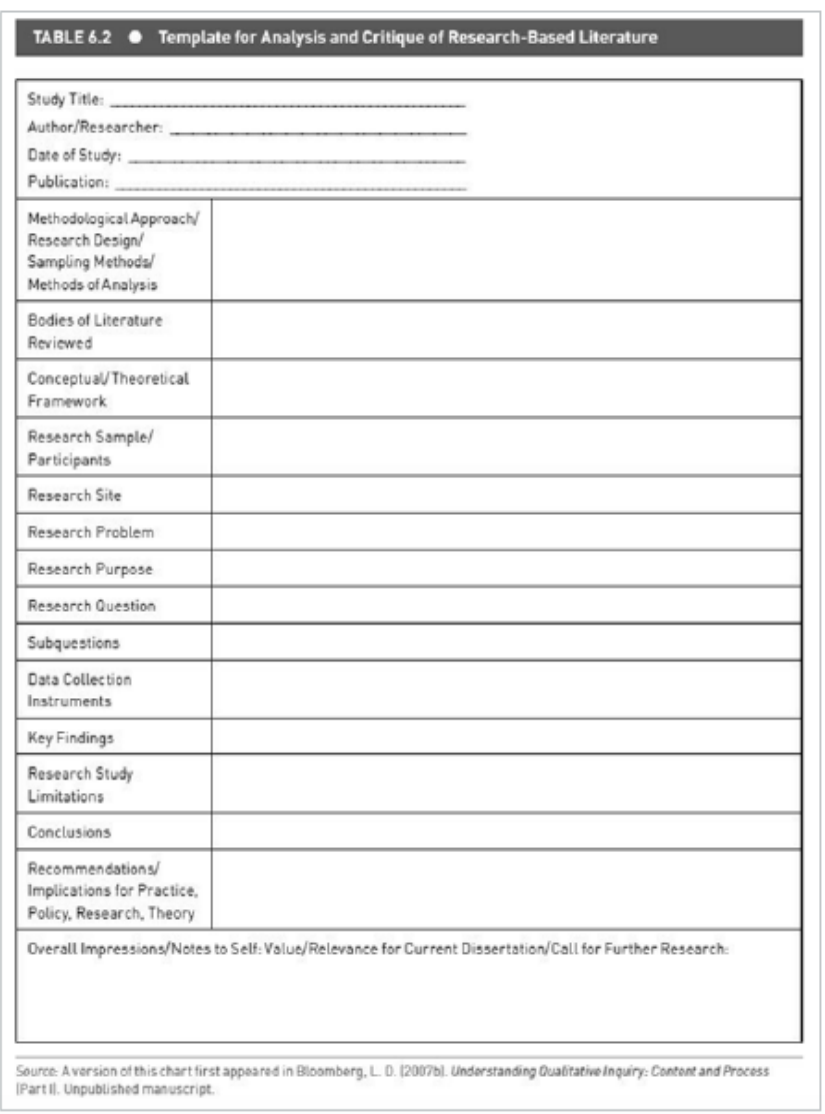

Figura 1. Uno de los dos esquemas propuestos por Bloomberg y Dale (2016) para analizar bibliografía basada en investigación. Fuente: Bloomberg, Dale 2016.

\section{TABLE 6.3 - Template for Analysis and Critique of Theoretical Literature}

\begin{tabular}{|c|c|}
\hline $\begin{array}{l}\text { Study Title: } \\
\text { Author/Researcher: } \\
\text { Date of Study: } \\
\text { Publication: }\end{array}$ & 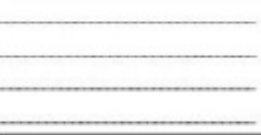 \\
\hline Overview of Theory & \\
\hline Key Premise/s & \\
\hline $\begin{array}{l}\text { Reasoning } \\
\text { Evidence is provided that } \\
\text { elearly supports the elaim/s. } \\
\text { Opposing elaims are } \\
\text { recognized and addressed. }\end{array}$ & \\
\hline $\begin{array}{l}\text { Relevance } \\
\text { Extent to which the information } \\
\text { directly supports your topic } \\
\text { and is useful to your study. } \\
\text { What ore the implications for } \\
\text { your current research? }\end{array}$ & \\
\hline $\begin{array}{l}\text { Overall Impressions/ } \\
\text { Evaluation } \\
\text { Does the author suggest the } \\
\text { findings can be opplied in } \\
\text { theory and/or practice? How } \\
\text { useful does this work seem to } \\
\text { you with regard to theoretical } \\
\text { and/or practical applications? }\end{array}$ & \\
\hline $\begin{array}{l}\text { Synthesis } \\
\text { Synthesize the pieces of your } \\
\text { critique to emphosize your own } \\
\text { main points about the author's } \\
\text { work; its relevance and/or } \\
\text { application to other the ories } \\
\text { you have reviewed and to your } \\
\text { own study. }\end{array}$ & \\
\hline
\end{tabular}

Figura 2. Una propuesta de esquema de análisis para artículos teóricos. Fuente: Bloomberg, Dale 2016.

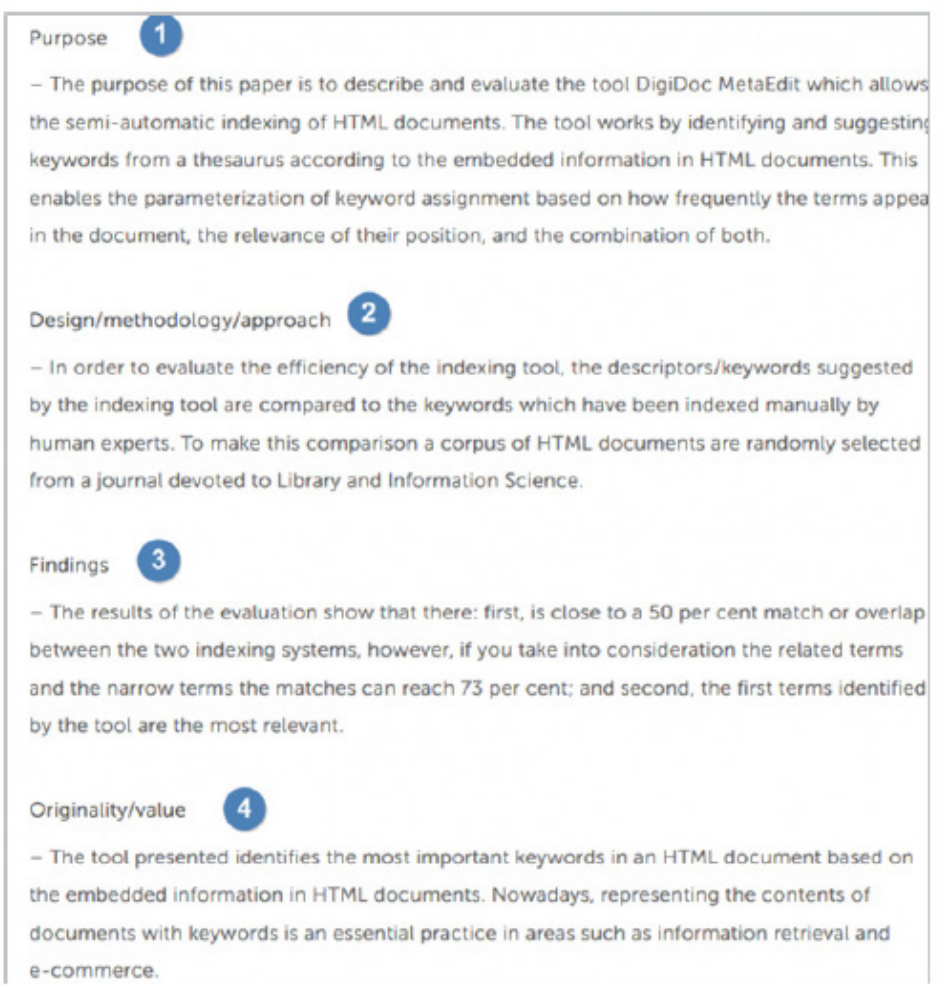

Figura 3. Ejemplo de aplicación del esquema de Emerald. Fuente: Vàllez, M. et al, 2015. (ver datos que siguen) 
En la Figura 3 puede observarse un ejemplo de utilización en un artículo de una publicación de Emerald Publishing.

Para posibles interesados, la referencia del artículo del que hemos tomado el ejemplo precedente es la que sigue:

Mari Vállez, Rafael Pedraza-Jiménez, Lluís Codina, Saúl Blanco, Cristòfol Rovira, (2015) "A semi-automatic indexing system based on embedded information in HTML documents", Library Hi Tech, Vol. 33 Issue: 2, pp.195-210, https://doi-org.sare.upf.edu/10.1108/LHT-12-2014-0114

\section{Procedimiento general}

A partir de los ejemplos anteriores, podemos ver que el procedimiento general para diseñar un esquema de análisis para tratar de forma sistemática un banco de documentos puede consistir entonces en los siguientes pasos:

1. Determinación de un esquema de análisis de acuerdo con los objetivos del proyecto. Puede utilizarse o adaptarse alguno de los esquemas generales como los vistos anteriormente, eventualmente, acompañados de elementos específicos según objetivos de la revisión.

2. Aplicación del esquema a todos y cada uno de los componentes del banco de documentos. El análisis mediante un esquema sistemático es muy potente, primero porque obliga a llevar a cabo una lectura profunda, y segundo porque obliga a la toma de notas observando siempre los mismos elementos. Esto hace aflorar relaciones, contradicciones, patrones, pautas, etc. que enriquecerán y aumentarán la capacidad crítica del autor de los análisis.

3. Comparación constante: eventualmente, la lectura de algunos de los documentos, sugerirá la conveniencia de volver a releer parte de algunos de los ya examinados anteriormente, lo que generará nuevas formas de interpretación, así como una óptima asimilación de los trabajos analizados.

4. Eventualmente, revisión del esquema. El análisis de los primeros documentos puede obligar o recomendar cambiar algún aspecto del esquema. En este caso, debe reformarse y aplicar los cambios que correspondan.

\section{Síntesis}

\section{Objetivos}

El objetivo de la fase de síntesis es producir un producto nuevo y distinto de la mera yuxtaposición de las partes componentes. Idealmente, la Síntesis debería consistir en un apartado con un alto valor añadido resultado de un esfuerzo intelectual en una triple dirección: la de agregar, pero también la integrar y la de interpretar.

Según Hart (2008), una revisión bibliográfica destinada a una tesis debe dar respuesta a todas o algunas de las preguntas como las siguientes:

- ¿Cuáles son las teorías y conceptos clave en el ámbito considerado?

- ¿Cuáles son las principales bases ontológicas y epistemológicas de la disciplina?

- ¿Cuáles son los temas y debates principales sobre el ámbito de estudio?

- ¿Cuáles son los principales problemas abordados por la disciplina?

- ¿Cómo se estructuran y organizan los conocimientos sobre la disciplina?

Nosotros añadiríamos, al menos una más:

- ¿Qué huecos u oportunidades de investigación ofrece el ámbito analizado?

Por su parte, Bloomberg y Dale (2016) señalan lo siguiente sobre la función de una revisión bibliográfica en una tesis :

The review of related literature involves the systematic identification, location, and analysis of material related to the research problem. This material can include books, book chapters, articles, abstracts, reviews, monographs, dissertations, research reports, and electronic media. A key objective of the literature review is to provide a clear and balanced picture of current leading concepts, theories, and data relevant to your topic or subject of study. The material, although consisting of what has been searched, located, obtained, and read, is not merely a simplistic summative description of the contents of articles and books, nor is it a series of isolated summaries of previous studies. Your readers are being asked to view this literature review as representing the sum of the current knowledge on the topic, as well as your ability to think critically about it.

Por su parte, Hart propone una visión sobre las aportaciones de una revisión que están basadas en un interesante grupo de preguntas, tal como muestra el diagrama de la figura 4.

El lector atento habrá observado que en la fase final, de Síntesis, recuperamos cuestiones examinadas en la primera entrega de esta serie. El motivo es que no se puede entender las características del producto final (la Síntesis) sin volver a considerar los objetivos de las revisiones bibliográficas que están al servicio de los 


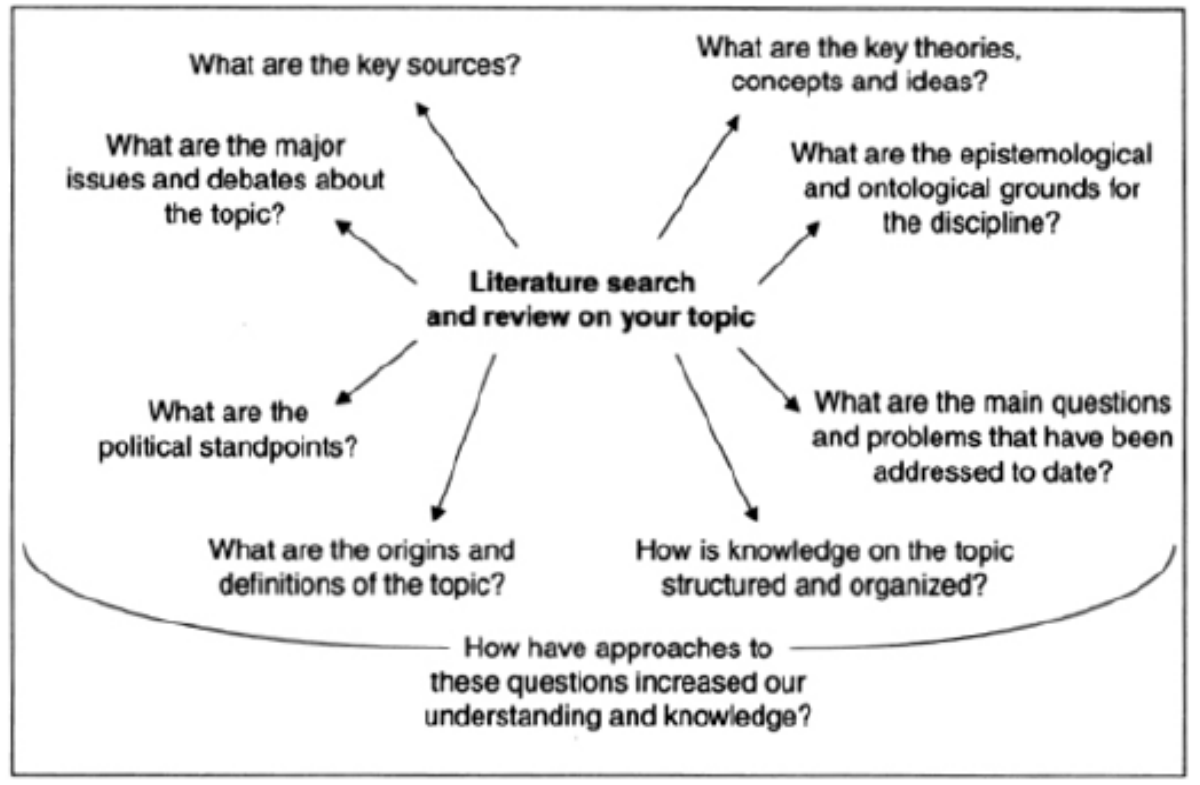

Figura 4. Diagrama de Hart (2008) sobre las aportaciones de un estado de la cuestión en forma de preguntas.

\begin{tabular}{|c|c|}
\hline Synthesis & Summary \\
\hline $\begin{array}{l}\text { An advanced reading technique or skill that requires } \\
\text { critical analysis, creativity, and insight. }\end{array}$ & A basic or intermediary reading technique. \\
\hline $\begin{array}{l}\text { Combines and contrasts information and ideas from } \\
\text { different sources. }\end{array}$ & Information is collated, reiterated, and restated. \\
\hline $\begin{array}{l}\text { Information from different sources is integrated to highlight } \\
\text { important points of connection and relatedness, to address } \\
\text { similarities and differences, and to draw conclusions. }\end{array}$ & $\begin{array}{l}\text { Information is pulled together and listed to hightight } \\
\text { important or key points. }\end{array}$ \\
\hline $\begin{array}{l}\text { Combines parts and elements from a variety of sources } \\
\text { into one unified or integrated entity. }\end{array}$ & $\begin{array}{l}\text { Addresses distinct sets of information. Each piece of } \\
\text { information or source remains distinct and separate. }\end{array}$ \\
\hline Focuses on deeper ideas and details. & Presents a cursory overview. \\
\hline $\begin{array}{l}\text { The final product reflects the author's knowledge } \\
\text { about the sources, but also creates new insights or } \\
\text { perspectives that add value to the intellectual discussion. }\end{array}$ & $\begin{array}{l}\text { The final product indicates and describes what the } \\
\text { sources stated. }\end{array}$ \\
\hline $\begin{array}{l}\text { Synthesis extends the literal meaning of a text to the } \\
\text { inferential level. The final product achieves new ways of } \\
\text { thinking and understanding about a body of literature. }\end{array}$ & $\begin{array}{l}\text { A summary captures the literal meaning of texts. The } \\
\text { final product demonstrates an understanding of the } \\
\text { overall body of titerature that was summarized. }\end{array}$ \\
\hline
\end{tabular}

Figura 5. Fuente: Bloomberg, Dale, 2016

estados de la cuestión.

\section{Agregar e interpretar}

En general, se considera que la síntesis de una revisión bibliográfica estará situada en algún punto de un continuo que tiene en un extremo una síntesis agregativa, dónde se agregan sin más los resultados de cada trabajo analizado, con mínima participación del autor de la revisión y con una síntesis interpretativa en el otro extremo, donde se produce una integración total de todos los resultados en una interpretación unificada.

En el contexto que nos ocupa, las síntesis están obligadas a situarse en punto algún intermedio, aunque siempre más cerca del extremo interpretativo que del meramente agregativo, manteniendo a la vez un equilibrio entre la necesidad de dar cuenta de los componentes del ámbito revisado, y la necesidad de llevar a cabo aportaciones propias que demuestren la capacidad del autor de la revisión para añadir valor a su trabajo de síntesis. 
Ello es debido a que los estados de la cuestión deben presentar el panorama más amplio posible pero a la vez, los evaluadores esperan que el autor de la revisión haya sido capaz de aportar una visión crítica propia, con capacidad de presentar elementos de relación, de contraste $\mathrm{y}$, al menos en algún grado de interpretación.

Además, la revisión debería servir tanto para identificar patrones como casos excepcionales, contradicciones entre hallazgos o entre teorías, identificar líneas de argumentación semejantes entre estudios diferentes, dar pie a nuevas hipótesis, justificar nuevas investigaciones, etc.

\section{Sintetizar y Resumir}

Bloomberg y Dale (2016) por su parte, se refieren a la misma dicotomía (Agregar vs Interpretar) con los conceptos de Summary y Synthesis, donde sumarizar correspondería a una operación parecida a la agregación y con menos requerimientos de esfuerzo intelectual, y menos valor añadido, mientras que la síntesis corresponde a un resultado muy superior, fruto a su vez, de un esfuerzo intelectual más intenso y mayor valor añadido (ver figura 5).

De nuevo, se espera que la fase de Síntesis de una revisión bibliográfica sistematizada se acerca más a una Synthesis que a un Summary.

\section{Conocer y argumentar}

Hart (2008, en Chap. 7 "Writing the Review") por su parte, presenta igualmente una dictomía parecida a las anteriores, categorizando los componentes que puede contener una buena revisión en dos tipos de aportaciones o elementos:

- Knowledge-based elements, o aportaciones basadas en el conocimiento

- Argumentational elements, o aportaciones basadas en argumentaciones

Los elementos basados en el conocimiento equivalen a la agregación o la operación de Summary. En concreto, Hart señala tres líneas de aportaciones en esta clase de componentes de una Síntesis:

- A description of previous work on the topic, identifying leading concepts, definitions and theories;

- Consideration of the ways in which definitions were developed and operationalized as solutions to problems seen in previous work;

- Identification and description of matters other researchers have considered important.
Por su parte, los elementos basados en la argumentación equivalen a la interpretación o la Synthesis. Entre esta clase de aportaciones, señala las siguientes:

- A description of what you find wrong in previous work on the topic;

- A proposal for action that might solve the problem your research;

- An explanation of the benefits that might result from adopting the proposal;

- A refutation of possible objections to the proposal.

\section{Agregar, integrar, interpretar}

Por su parte, Noblit y Hare (1988) proponen la tríada agregar, integrar, interpretar como los principales integrantes de una síntesis. La distribución de papeles de cada una de estas acciones es la siguiente:

- Agregar corresponde a la mera yuxtaposición de resúmenes de lo que dice cada trabajo, del estilo, Fulano afirma tal cosa, Zutano tal otra, y Merengano, tal otra.

- Integrar implica unir de alguna forma los resultados de dos o más investigaciones. Esta unión puede tener la forma de una propuesta teórica o una síntesis de ideas de diferentes trabajos. Se puede expresar en forma textual, esto es de discurso, o en forma de tablas o diagramas.

- Interpretar representa el grado superior de síntesis, ya que corresponde a contextualizar, comparar, valorar, criticar, etc., los diversos trabajos objeto de síntesis. Es una opción solamente al alcance de autores o de equipos que han obtenido un buen grado de conocimiento y de madurez en el ámbito en que llevan a cabo su trabajo de revisión.

Según esta distribución, la síntesis ideal combina los tres componentes en algún grado. Toda síntesis, por el mero de serlo, tiene algo de agregación, pero también debería tener integración de aquellos aspectos que lo justifiquen, y algo de interpretación para honrar el hecho de que una buena síntesis debe ser algo más que la suma de las partes.

\section{Síntesis narrativas}

Las síntesis narrativas son la forma más habitual en el caso de los estados de la cuestión para trabajos académicos o tesis doctorales. También son útiles como parte de los antecedentes de un artículo científico, y por supuesto, pueden ser el formato idóneo para un trabajo de revisión autocontenido en el ámbito de las Ciencias Humanas y Sociales. 
Para elaborar síntesis narrativas se pueden utilizar diversas aproximaciones (Noblit y Hare, 1988; Sandelowski y Barroso, 2007; Hart, 2008), pero tres de las más útiles pueden ser las siguientes:

- Metasíntesis. Para sintetizar trabajos de naturaleza relativamente unitaria o monográfica. Ejemplo: sintetizar artículos publicados sobre una determinada intervención, como, p.e., "la perspectiva de género en planes de estudio en las universidades" (Noblit y Hare, 1988).

- Síntesis por facetas o subtemas. Para trabajos multidisciplinares. P.e. sobre un área o una subárea de conocimiento, p.e., "SEO y medios de comunicación"

- Cronologías. Para enfoques historicistas, p.e., "evolución la infografía en medios de comunicación"

\section{Metasíntesis}

Según Noblit y Hare (1988), los trabajos reunidos para una revisión pueden tener cuatro tipo de relaciones:

- Pueden tratar temas diferentes

- Puede estudiar temas similares

- Pueden ser trabajos que se refuten entre ellos

- Pueden ser estudios que ayuden a constituir líneas de argumento

Respecto a la primera relación, como es lógico, descartan que se puedan sintetizar: si no tienen nada en común, no tiene sentido intentar unirlos. Esto nos deja con las otras tres relaciones, que dan lugar a las tres clases de síntesis que proponen:

- Traducción recíproca: dos o más trabajos usan los mismos conceptos, ideas, teorías, etc., pero utilizan diferentes denominaciones o las enfocan desde diferentes perspectivas o diferentes tradiciones teóricas. El trabajo de síntesis consiste en poner de relieve esta traducción recíproca.

- Síntesis de confrontación: consiste en identificar dos o más trabajos que presentan ideas contrapuestas, o al menos suficientemente diferenciadas sobre el mismo fenómeno u objeto de estudio como para representar ideas que se refutan entre ellas, totalmente o en parte.

- Líneas de argumento: dos o más trabajos pueden presentar argumentos complementarios y coincidentes, lo que nos permite encontrar una línea argumental coherente entre diversos trabajos que ayude a construir una aportación teórica o a establecer un panorama coherente de un ámbito determinado, de una propuesta teórica, etc.

\section{Facetas y cronologías}

La síntesis por facetas y cronologías consisten en estructurar los diversos trabajos objetos de revisión por subtemas o facetas del área o del ámbito estudiado, o bien por hitos o momentos cronológicos.

En realidad, el tratamiento por facetas y por líneas de tiempo es una de las formas habituales en las que se suelen presentar los estados de la cuestión.

Como sea, tanto la metasíntesis, como las facetas y cronologías permiten realizar las tres operaciones fundamentales de una síntesis: la agregación, la integración y la interpretación.

\section{Propuesta de procedimiento general}

Para esta propuesta de procedimiento para una síntesis narrativa, partimos de la siguiente situación:

- - Suponemos que se han llevado a cabo las fases de Búsqueda y Evaluación (la SAL de SALSA), lo cual ha producido un banco de documentos.

- - Suponemos que se ha llevado a cabo el Análisis (la segunda A en SALSA) mediante algún esquema que ha producido entre otros resultados resúmenes informativos de cada trabajo del banco de documentos. Estos resúmenes pueden tener extensiones diferentes según cada trabajo analizado, pero podemos suponer unas 300 palabras por cada uno en promedio.

- Esto nos deja pendiente la Síntesis (la segunda S en SALSA), cosa de la que nos ocupamos ahora.

A partir de este escenario, los pasos a ejecutar para disponer de una síntesis de tipo narrativo son los siguientes:

1. Revisar los parámetros del proyecto o trabajo de investigación, en particular objeto de estudio, objetivos y preguntas de investigación para el cual se está desarrollando la revisión sistematizada.

2. Revisar el conjunto de los resúmenes obtenidos en las fase de Análisis y ordenar los resúmenes por alguno de los criterios anteriores: metasíntesis, facetas o cronología, o cualquier otro criterio según la naturaleza de la investigación.

3. Revisar y editar los resúmenes de cada trabajo, uno por uno, para que adquieran un estilo coherente y unitario.

4. Aplicar procedimientos de agregación, integración o interpretación al conjunto de trabajos según corresponda. Redactar frases de transición entre los diferentes párrafos. Atribuir con cuidado las ideas a cada autor. 
5. Señalar claramente los fragmentos literales y marcarlos de forma conveniente en el cuerpo de la síntesis como tales citas literales.

6. Volver a revisar el conjunto del trabajo y velar por su integridad de estilo y por su coherencia. Extremar las precauciones para evitar malas prácticas en relación con la debida atribución de las ideas a cada autor, y en todo caso, evitar el más mínimo riesgo de plagio.

7. Considerar añadir tablas y diagramas como elementos superiores de síntesis e integración .

8. Volver a retomar la síntesis (mejor dejar transcurrir uno o dos días), revisarla de nuevo forma global y asegurar la coherencia del conjunto, editando, añadiendo información, mejorando los elemento de transición, etc.

\section{Producto final}

La fase de Síntesis es la fase final de una revisión sistematizada, pero esta síntesis puede adoptar diversas formas como producto o presentación final. En el contexto en el que nos movemos, probablemente será parte de un trabajo más amplio, como hemos señalado varias veces. En tal caso, constituirá el núcleo del estado de la cuestión.

$\mathrm{Si}$, en cambio, está destinada a ser un trabajo de revisión independiente, deberá tener una introducción, con indicación de objeto de estudio y preguntas de investigación, como cualquier otra investigación. Siendo en este caso, la síntesis el resultado y los procedimientos del framework SALSA el método utilizado.

En este caso, deberá tener también una discusión y unas conclusiones, con tal de completar, mediante una estructura tipo IMRyD, el formato que debe tener toda investigación completa.

En los puntos anteriores, hemos señalado la conveniencia de considerar el uso de tablas y diagramas como forma superior de síntesis. En lo que sigue, vamos a considerar someramente estos dos componentes. Para esto, recurriremos a un trabajo nuestro anterior más amplio sobre el tema (que el lector interesado puede consultar aquí).

\section{Tablas}

Una tabla o matriz se compone de una serie de filas y columnas. El cruce de las mismas produce las celdas, que contienen valores. Esta simple estructura es una poderosa formas de sintetizar información, y de mostrar relaciones, ya se trate de datos cualitativos o cuantitativos.
Hay pocas reglas sobre cómo construir tablas a los efectos que nos interesan aquí, pero desde luego disponemos de algunas.

La primera nos dice que, cuando estamos representando entidades y sus características o propiedades, conviene proceder de este modo:

\section{- Las entidades son las filas}

\section{- Las propiedades son las columnas}

La segunda y no menos simple regla es que debemos usar un número limitado de columnas. En concreto, algunos autores indican que las tablas (para estos usos) no deberían tener más de una docena de columnas, "aunque seis o siete es más manejable" (Miles, Huberman, y Saldaña 2014).

El número de filas es mucho menos conflictivo, pero es evidente que si las usamos como medio para sintetiza resultados, no deberían ir más allá de unas pocas decenas.

La tercera regla, nos dice que las tablas deben tener:

- Un título, preferentemente precedido de una numeración

- Un pie donde se informe de la fuente y/o de cualquier otro dato que ayude a interpretarla

La cuarta, nos dice que las tablas pueden estar ordenadas, cosa que se puede llevar a cabo mediante la selección de una de las columnas que consideremos más significativas como eje de tal ordenación. Esto nos puede permitir tener varias tablas con los mismos datos pero diferentes ordenaciones, lo cual ayuda a la visualización de la información.

En la figura 6 vemos un caso ejemplar de utilización de tablas para sintetizar resultados. A los autores, la tabla que ocupa un total de 2 páginas, con un total de 6 columnas y 14 filas, no solamente les sirvió para explicar y comparar, sino también para argumentar y llevar al lector a la conclusión deseada por los autores. Al proponer la comparación de las propiedades de cada una de las categorías, les permitió reforzar y apoyar de forma muy sólida sus conclusiones.

Otro ejemplo de uso de tablas para sintetizar diversos resultados procedentes de un trabajo de revisión lo podemos ver en el artículo de Olarte-Mejía y Ríos-Osorio (2015) (Figura 7).

\section{Diagramas}

Un diagrama es cualquier clase de gráfico que se utiliza para representar ideas. Que sepamos, no existe una lista cerrada de tipos de diagramas, aunque desde luego hay excelentes taxonomías que intentan poner 


\begin{tabular}{|c|c|c|c|c|c|}
\hline \multirow[b]{2}{*}{ Label } & \multirow[b]{2}{*}{ Description } & \multicolumn{4}{|l|}{ Methods used (SALSA) } \\
\hline & & Seanch & Apprasisal & Synthesis & Analysis \\
\hline Cribcal review & 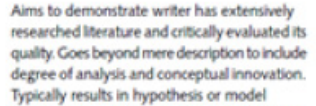 & $\begin{array}{l}\text { Seedis to identify } \\
\text { most significant items } \\
\text { in the field }\end{array}$ & $\begin{array}{l}\text { No formal quality } \\
\text { assessment Aattempts } \\
\text { to evaluaste according } \\
\text { to contrilibution }\end{array}$ & $\begin{array}{l}\text { Typically narrative. } \\
\text { pethaps conceptial } \\
\text { or chronological }\end{array}$ & $\begin{array}{l}\text { Sigrificant component: seeds to } \\
\text { identify concepptual contribution } \\
\text { to embody exating or derive } \\
\text { new theory }\end{array}$ \\
\hline Laenature review & 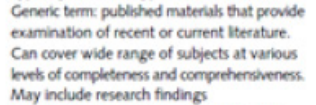 & $\begin{array}{l}\text { May or may not } \\
\text { incluse comprethenive } \\
\text { seaching }\end{array}$ & $\begin{array}{l}\text { May or may not } \\
\text { include quility } \\
\text { asssesment }\end{array}$ & Typically narative & $\begin{array}{l}\text { Anayysis may be chronological, } \\
\text { conceptual, thematcic, etc. }\end{array}$ \\
\hline $\begin{array}{l}\text { Mapping review/ } \\
\text { systemabc map }\end{array}$ & 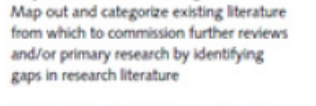 & $\begin{array}{l}\text { Completeness of } \\
\text { searching determined } \\
\text { by time/scope } \\
\text { constraints }\end{array}$ & $\begin{array}{l}\text { No formal quality } \\
\text { sasessment }\end{array}$ & $\begin{array}{l}\text { May be graphical } \\
\text { and tabular }\end{array}$ & $\begin{array}{l}\text { Characterzes quantity and } \\
\text { quality of literature, perhups by } \\
\text { study design and other key } \\
\text { features. May idently need for } \\
\text { primary or secondary reseasch }\end{array}$ \\
\hline Meta-analysis & $\begin{array}{l}\text { Technique that statistically combines the } \\
\text { results of quantintive studies to provide a } \\
\text { moce precise effect of the results }\end{array}$ & $\begin{array}{l}\text { Aims for exhusustive. } \\
\text { compechensive searching. } \\
\text { May use funnel plotot to } \\
\text { assess comoleteness }\end{array}$ & $\begin{array}{l}\text { Quality assessment may } \\
\text { determine inclusion/ } \\
\text { exclusion and/or } \\
\text { sensaviver andives }\end{array}$ & $\begin{array}{l}\text { Graphical and } \\
\text { tabular with } \\
\text { nusrative commentary }\end{array}$ & $\begin{array}{l}\text { Numerical anulysis of measures } \\
\text { of effect assuming absence of } \\
\text { beterogenefity }\end{array}$ \\
\hline
\end{tabular}

Figura 6. Un excelente ejemplo de utilización de tablas para explicar y comparar. Fuente: Grant y Booth, 2009. Clic para zoom.

\begin{tabular}{|c|c|c|}
\hline \multicolumn{3}{|c|}{$\begin{array}{l}\text { Tabla } 2 \\
\text { Relación de enfoques y estrategias de la rsu }\end{array}$} \\
\hline Enfoque & Estrategias & Autor \\
\hline \multirow{7}{*}{ Humanista } & $\begin{array}{l}\text { Reonentación del modelo de traneporte y } \\
\text { movilización en el campus. }\end{array}$ & $\begin{array}{l}\text { Atherton y Guruco, 2011; Hancock y } \\
\text { Nuttman, 2014. }\end{array}$ \\
\hline & $\begin{array}{l}\text { Orientación de politicas hacia campus } \\
\text { verdes, sosteribles. }\end{array}$ & $\begin{array}{l}\text { Savelyeva y McKenna, 2011; Atherton y } \\
\text { Giuro, 2011; Hancock y Nuttman, 2012; } \\
\text { Goni et at, } 2013 \text {. }\end{array}$ \\
\hline & $\begin{array}{l}\text { Relacionamiento con las comuridades } \\
\text { (regionales, nacionales, locales). }\end{array}$ & Atherten $y$ Gurco, 2011; Dima ef al, 2013. \\
\hline & $\begin{array}{l}\text { Contribución a causas humanitarias (hacer } \\
\text { filantropia, voluntariado). }\end{array}$ & $\begin{array}{l}\text { Olberding, 2012: Bryant et al, 2012; Ganga } \\
\text { y Navarrete, 2012. }\end{array}$ \\
\hline & $\begin{array}{l}\text { Abordar la dimensión de ss con empleados, } \\
\text { y sus famvilias. }\end{array}$ & Ganga $y$ Navarrete, 2012 \\
\hline & $\begin{array}{l}\text { Implementax procesos de recompensa a } \\
\text { docentes en el ambito personal y familiax. }\end{array}$ & Reybold, 2003. \\
\hline & $\begin{array}{l}\text { Vinculación de estudiantes en prácticas } \\
\text { co-curriculares, de trabajo comunitario. }\end{array}$ & $\begin{array}{l}\text { Aronson y Webstex, } 2007 \text {; Antunes y Marti- } \\
\text { nez, 200s; Savelyeva y McKenna, } 2011 .\end{array}$ \\
\hline \multirow{9}{*}{ Pedagógice } & $\begin{array}{l}\text { Apropiar la sostentbilidad en la enserianza } \\
\text { y aprendizaje. }\end{array}$ & Atherten y Gurco, 2011. \\
\hline & $\begin{array}{l}\text { Inculcar el compromiso de sostenibilidad } \\
\text { desde la Investigacion. }\end{array}$ & Atherton y Gurco, 2011: Goni et at, 2013. \\
\hline & $\begin{array}{l}\text { Incluir formación en Desarnollo Sostenible- } \\
\text { to en el curriculo de algunos programas. }\end{array}$ & $\begin{array}{l}\text { Savelyeva y McKenna, 2011: Rusinloo, 2010; } \\
\text { Ganga y Navarrete, } 2012 .\end{array}$ \\
\hline & $\begin{array}{l}\text { Implementación de estrategia de enserian. } \\
\text { za en filantropía. }\end{array}$ & Olberding, 2012 \\
\hline & $\begin{array}{l}\text { Formación de los estudiantes hacia la } \\
\text { compensación para con la sociedad e insti. } \\
\text { tución (filantropia de caridad, donación). }\end{array}$ & $\begin{array}{l}\text { Savelyeva y McKenna, 2011: Olberding } \\
\text { 2012. Almeida of al., (2013) }\end{array}$ \\
\hline & $\begin{array}{l}\text { Inclusión de estudiantes en la configura. } \\
\text { ción de Planes de Estudio. }\end{array}$ & $\begin{array}{l}\text { Savelyeva y Mckienua, 2011: Olberding, } \\
\text { 2012; Dima ct a1., } 2013\end{array}$ \\
\hline & $\begin{array}{l}\text { Formar estudiantes en desarrollo, desarro- } \\
\text { llo sostenible, impacto (s) ambiental (es) y } \\
\text { social (es). }\end{array}$ & $\begin{array}{l}\text { Barth et al, 2014: Almeida et al,2013; Marti- } \\
\text { nez et af., 2013; Barth et at., } 2014 .\end{array}$ \\
\hline & Educar en la ideo-politica del Estado. & Aroncon y Webstex, 2007: Lai y Lo, 2011. \\
\hline & $\begin{array}{l}\text { Articular los intereses de los estudiantes a } \\
\text { la diversidad intencional de la institución. }\end{array}$ & Arizabaleta, 2006: Lee et at, 2012 \\
\hline
\end{tabular}

Continúa...

Figura 7. Fuente: Olarte-Mejía y Ríos-Osorio 2015. (Vemos aquí solamente una parte de la tabla). Clic para descargar el artículo en pdf 
un poco de orden sobre ellas. No obstante, los más conocidos son las que se refieren a los diagramas para visualizar estadísticos (cosa que no trataremos aquí).

Por tanto, con los diagramas tenemos una situación, afortunadamente, parecida a las tablas: no existe a priori, ninguna limitación sobre el tipo de cosas que podemos expresar de forma gráfica. Esto no impide que, tipos de diagramas muy bien asentados, como los diagramas de Gantt, los diagramas de flujo o los mapas conceptuales no tengan algunas reglas, por más sencillas que sean.

La cuestión es que, si los comparamos con las tablas, el uso que suele darse a los diagramas está más vinculado a representar procesos y relaciones de influencia. Pero ni siquiera esto limita su uso real. Nada nos impide expresar con un diagrama cualquier clase de constructo. La condición previa ineludible es tener una buena conceptualización del mismo.

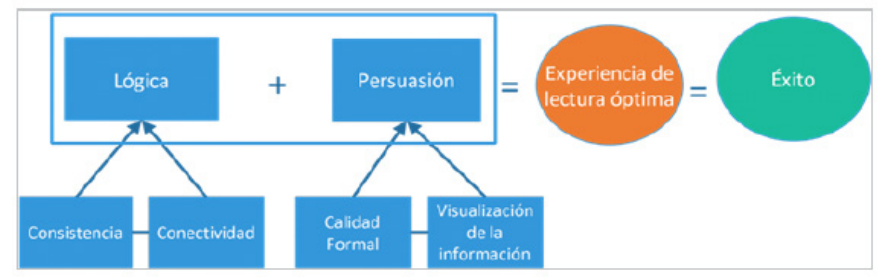

Figura 8 Un ejemplo de constructo que incluye relaciones de influencia, en este caso, en el ámbito de la calidad en trabajos académicos, expresado mediante un diagrama que representa los dos componentes principales de calidad con subcomponentes. Fuente: elaboración propia

\section{Mapas conceptuales}

Un mapa conceptual es una clase de diagrama que intenta representar de forma gráfica tanto los componentes de un sistema conceptual como las relaciones entre ellos.

Por sistema conceptual podemos entender al menos dos cosas:

- Cualquier conjunto más o menos coherente y organizado de ideas en el seno de una conceptualización más amplia o de una teoría más o menos compleja, por ejemplo, la teoría de la evolución;

- Cualquier conjunto de entidades y sus relaciones, por ejemplo, las empresas del sector de la comunicación de un determinado país.

Los mapas conceptuales no tienen ningún ámbito de restricción, de manera que cualquier aspecto del mundo real o del mundo conceptual es susceptible de ser representado en un diagrama de esta clase.

La complejidad del sistema que debe ser representado tampoco establece ningún tipo de restricción de entrada: podemos representar con un mapa conceptual una teoría tan compleja o simple como queramos (o podamos).

Los mapas conceptuales se pueden utilizar para construir modelos de una gran cantidad de situaciones o aspectos de la realidad, desde sistemas de carretas hasta mapas de sitios web, pasando por relaciones de parentesco, vínculos entre grupos de empresas o relaciones entre componentes de un marco teórico (de aquí, su uso en revisiones sistemáticas).

\section{Procesos y relaciones de influencia}

Otro tipo de diagrama clásico es la representación de procesos, o de factores de influencia. Si utilizamos los dos componentes que ya conocemos, los nodos y los arcos, resulta fácil sintetizar esta clase de aspectos (siempre que tengamos algunas claras al respecto).

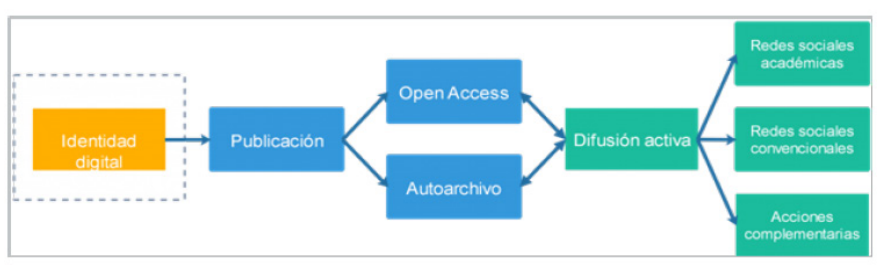

Figura 9. Ejemplo de diagrama que muestra un proceso: SEO académico. Fuente: elaboración propia.

\section{Aportación polifuncional}

Si el trabajo de revisión se ha llevado a cabo para presentar un estado de la cuestión, cabe señalar que de estos se espera mucho porque cumple diversas funciones.

Por un lado, es una demostración de que su autor ha alcanzado la madurez intelectual y académica suficiente como para sintetizar trabajos de otros autores y presentar un cuadro cohesionado de un ámbito o, con frecuencia de la intersección de varias disciplinas.

Pero, por otro lado, de un estado de la cuestión se espera que ofrezca un retrato certero y fiel del campo de estudios o de una intersección de disciplinas.

El mejor estado de la cuestión será entonces el que consiga combinar los tres componentes de agregación, integración e interpretación de una forma armoniosa, dado garantías de solvencia, transparencia y rigor, y proporcionado a la vez un producto creativo, sugerente, rico y fértil al ser capaz de abrir y mostrar nuevos caminos, así como detectar tendencias y oportunidades de investigación. 
Si la síntesis se ha producido como un trabajo de revisión, esto es, como un proyecto de investigación independiente, necesitará documentar con sumo cuidado y detalle las fases con las que se ha llevado a cabo, además de integrar la síntesis en una estructura más amplia, tipo IMRyD a fin de dotar al resultado final del contexto completo de una investigación.

\section{Resumen: el Framework ReSiste-CHS en dos diagramas}

Dado que defendemos aquí el uso de diagramas (y de tablas) para sintetizar conceptos (y resultados), vamos a mostrar el Framework ReSiste-CHS que nos ocupado a lo largo de estas entregas, en dos versiones: compacta y extendida (Figuras 10 y 11).

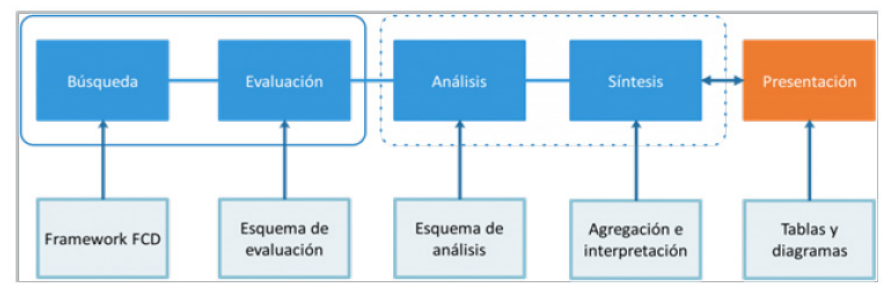

Figura 10. Versión compacta del Framework ReSiste-SHC en forma de diagrama. Las dos primeras fases aparecen enmarcadas porque son las que admiten un mayor grado de homogeneización. Las fases siguientes también están enmarcadas por una línea de puntos porque los criterios y procedimientos están vinculados al proyecto concreto.

Una vez mostrada la versión precedente, vamos ahora a ver el diagrama con dos componentes adicionales hechos explícitos (figura 11).

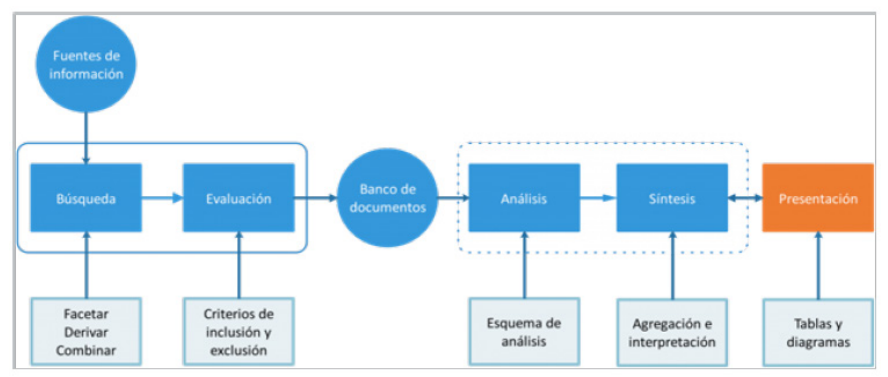

Figura 11. Versión extendida del Framework ReSiste-CSH haciendo explícitos los componentes Fuentes de información y Banco de documentos, y asignando rótulos más explicativos a las fases componentes. Fuente: elaboración propia.

\section{Balance}

¿Hasta qué punto es sistemática una revisión sistematizada? Llegados a este punto, nos gustaría presentar un resumen/balance con relación a lo que hemos

visto en los tres artículos que componen esta serie mediante una serie de tablas.

Tabla 1: Tipos de trabajo, objetivos y relación con los tipos de revisiones

\begin{tabular}{|c|c|c|} 
Tipo & \multicolumn{1}{c}{ Objetivos } & $\begin{array}{c}\text { Tipo de } \\
\text { revisión más } \\
\text { adecuada }\end{array}$ \\
\hline $\begin{array}{c}\text { Trabajos de } \\
\text { revisión }\end{array}$ & $\begin{array}{l}\text { Evaluar la eficacia de un } \\
\text { tratamiento o de una } \\
\text { intervención que afecta a } \\
\text { una población determinada } \\
\text { (p.e. niños, o inmigrantes, o } \\
\text { personas que han sufrido un } \\
\text { determinado trauma, etc.) }\end{array}$ & Sistemática \\
\hline Estados de & $\begin{array}{l}\text { Determinar la situación, las } \\
\text { principales teorías, tenden- } \\
\text { cias y problemas de un } \\
\text { la cuestión } \\
\text { ámbito del conocimiento. } \\
\text { ldentificar huecos y oportu- } \\
\text { nidades de investigación. }\end{array}$ & \\
\hline & Sistematizada \\
\hline
\end{tabular}

Una vez vista la relación anterior, en la siguiente tabla nos preguntamos cuánto de sistemático hay en una revisión sistematizada, siempre en el contexto de estados de la cuestión para trabajos académicos y memorias de proyectos de investigación. Tabla 2: qué es sistemático en una revisión
sistematizada

Fase

Búsqueda

Evaluación

Análisis

Síntesis

\section{Grado de sistematización}

Muy alto. Comparte criterios idénticos con las revisiones sistemáticas.

Medio. Comparte una misma filosofía con las revisiones sistemáticas, pero no procedimientos concretos, ya que estos son dependientes de los objetivos y características de cada revisión y de su contexto y tipo de investigación.

Por su parte, la tabla siguiente, muestra un resumen de algunos de los tipos principales de revisiones bibliográficas y su relación con las revisiones sistemáticas o sistematizadas.

Tabla 3: tipos de revisiones y relación con la aproximación sistemática/sistematizada

$$
\text { Tipo }
$$

Trabajos de revisión de intervenciones médicas, programas o políticas sociales que afectan a una población bien determinada

Estados de la cuestión como parte de trabajos académicos

\section{Sistemática Sistematizada}

Sí

NO sí 


\begin{tabular}{l|l|l|}
\hline $\begin{array}{l}\text { Estados de la cuestión para } \\
\text { memorias de investigación } \\
\text { (solicitud de financiación) }\end{array}$ & NO & Sí \\
\hline $\begin{array}{l}\text { Trabajos de revisión en } \\
\text { el ámbito de las Ciencias } \\
\text { Humanas y Sociales, como } \\
\text { trabajos autocontenidos }\end{array}$ & NO & Sí \\
\hline $\begin{array}{l}\text { Estados de la cuestión como } \\
\text { parte de artículos cientí- } \\
\text { ficos (antecedentes) }\end{array}$ & NO & Sí \\
\hline
\end{tabular}

La tabla precedente muestra la adecuación de cada tipo de revisión, sistemática o sistematizada, según el tipo de investigación y los objetivos de la misma.

Por último, la tercera tabla muestra el grado de aplicación del Framework ReSiste-SHC según el objetivo de la revisión y su formato final:

Tabla 4: Framework ReSiste-CSH: ¿dónde podemos aplicar cada fase?

Escenarios de máxima
aplicabilidad (Búsqueda,
Evaluación, Análisis y
Síntesis)
- Estados de la cuestión como
parte de trabajos de final
de máster, tesis doctorales
y memorias para solicitar
financiación de proyectos en
planes nacionales o similares.
- Trabajos de revisión que se
presentan como trabajos
independientes o autocon-
tenidos. Es decir, como
investigaciones completas
en sí mismas, que cumplen
el estándar IMRyD.

Escenarios de aplicabilidad parcial (Búsqueda y Evaluación)
La tabla precedente resume algo en lo que hemos insistido en otros apartados, el Framework ReSiste-SCH está concebido para su aplicación a estados de la cuestión destinados a formar parte de trabajos académicos más amplios, en cuyo caso, tiene sentido aplicar las cuatro fases.

En la misma situación se encuentran los trabajos de revisión en el ámbito de las Ciencias Humanas y Sociales. Precisamente por aspirar a ser consideradas investigaciones en sí mismas. deben aplicar las cuatro fases, con lo cual tales trabajos se ajustan al estándar IMRyD. Esto es, presentan unos resultados (la síntesis) gracias a la aplicación de unos métodos que se han quedado documentado a un conjunto de datos (el banco de documentos).

Además, se pueden aplicar al menos las dos primeras fases (Búsqueda y Evaluación) a otros usos vinculados con los estados de la cuestión, aunque sea en versión más ligera, como los antecedentes en artículos académicos.

\section{Conclusiones}

En esta serie de tres capítulos hemos examinado los fundamentos de las revisiones bibliográficas basadas en aproximaciones sistemática, o más simplemente, de las revisiones sistematizadas.

Hemos presentado el grupo principal de obras académicas en las que nos hemos basado para presentar un marco o propuesta de trabajo concreto que hemos denominado Framework Resiste-CHS.

Después, y siguiendo a su vez otro marco de referencia, el Framework SALSA hemos presentado las 4 fases en las que se desarrolla nuestra propuesta: Búsqueda, Evaluación, Análisis y Síntesis.

En este sentido, hemos visto que, mientras las dos primeras fases, Búsqueda y Evaluación, son (relativamente) fáciles de homologar para casi cualquier clase de revisión sistematizada, las otras dos, Análisis y Síntesis, son estrechamente dependientes del tipo de revisión y de sus objetivos.

No obstante, incluso de estas dos fases es posible aportar algunos elementos de sistematización que ayuden al rigor y la calidad de la revisión, justificando de paso el término sistematizada que se predica de esta clase de revisiones.

Por último, debemos señalar que no solamente las revisiones sistematizadas pueden producir buenos estados de la cuestión. Es evidente que la experiencia y el conocimiento de un investigador senior pueden bastar para producir piezas de enorme calidad en este terreno.

Sin embargo, en nuevos proyectos y en aquellos casos que se necesitan para misiones críticas, tales como trabajos académicos (donde hay títulos académicos en juego) o memorias de proyectos (donde hay financiación en juego) tiene poco sentido no llevarlas a cabo con las mejores garantías posibles. A cambio de un proceso más exigente, obtendremos resultados mucho más solventes y con muchas más probabilidades de éxito.

\section{Referencias}

En esta tercera entrega hemos fusionado las otras dos listas de referencias con la nuevas que hemos añadido para esta, de manera que proporcionamos ahora una única lista unificada.

Alana James E; H. Slater, Tracesea. Writing your Doctoral 
Dissertation or Thesis Faster. London: Sage, 2014.

Barnett-Page, E.; James Thomas, J. "Methods for the synthesis of qualitative research: a critical review". BMC Medical Research Methodology 2009, 9:59 doi:10.1186/14712288-9-59 [http://www.biomedcentral.com/1471$2288 / 9 / 59]$

Bell, Judith ; Waters, Stephen. Doing your Research Project: A Guide for first-time Researchers. Berkshire: McGraw Hill, 2014.

Blaxter, Loraine; Hugues, Christina ; Tight, Malcolm. How to Research. Berkshire: McGraw Hill, 2010.

Bloomberg, Linda D.; Volpe, Marie. "Developing and Presenting Your Literature Review". En: Completing your Qualitative Dissertation: A Road Map From Beginning to End. London: Sage, 2016.

Boland, Angela; Cherry, M. Gemma; Dickson, Rumona. Doing a Systematic Review: A Student's Guide. London: Sage, 2014.

Booth, Andrew; Papaionnou; Sutton, Anthea. Systematic Approaches to a Successful Literature Review. London: Sage, 2012.

Codina, Lluís. "Bases de datos académicas para investigar en Comunicación Social: revisiones sistematizadas, grupo óptimo y protocolo de búsqueda". Lecciones. Portal de la Comunicación InCom-UAB. Julio 2017. Acceso: http://www. portalcomunicacion.com/lecciones_det.asp?id=96

Codina Lluís. Revisiones bibliográficas sistematizadas: procedimientos generales y Framework para ciencias humanas y sociales. Barcelona: Universitat Pompeu Fabra, Departamento de Comunicación, Máster Universitario en Comunicación Social; 2018. 87 p.

Dixon-Woods, Mary et al. "Conducting a critical interpretive synthesis of the literature on access to healthcare by vulnerable groups". BMC Medical Research Methodology, July 2006 v. 6, n. 35. https://doi.org/10.1186/1471-2288-6-35

France, E.F. et al. "Protocol-developing meta-ethnography reporting guidelines (eMERGe)". BMC Medical Research Methodology. 2015, 15:103. [https://bmcmedresmethodol. biomedcentral.com/articles/10.1186/s12874-015-0068-0]

Fink, Arlene. Conducting Research Literature Review. London: Sage, 2014.

Guirao, Silamani J. Adolf. "Utilidad y tipos de revisión de literatura". Ene: Revista de Enfermería. Enero 2015 v. 9, n. 2 https://dx.doi.org/10.4321/S1988-348X2015000200002

Gouch, David; Oliver, Sandy; Thomas, James. An Introduction to Systematics Reviews. London: Sage, 2012.

Grant, Maria J.; Booth, Andrew. "A typology of reviews: an analysis of 14 review types and associated methodologies". Health Information and Libraries Journal, 26, pp.91-108, 2009. Acceso: onlinelibrary - wiley

Hart, Christopher. Doing a Literature Review: Releasing the Social Science Research Imagination. London: Sage, 2008

Jesson, Jill K.; Matheson, Lydia; Lacey, Fiona M. Doing your Literature Review: Traditional and Systematic Techniques. London: Sage, 2011.
Machi, Lawrence A.; McEvoy, Brenda T. The Literature Review: Six Steps to Success. Thousand Oaks, California: Corwin, 2012.

Major, Claire H.; Savin-Baden, Maggi. An introduction to Qualitative Research Synthesis: Managing the Information Explosion in Social Science Research. New York: Routledge, 2010.

Miles, M.B; Huberman, M.A; Saldaña, J. Qualitative Data Analysis: A Method Sourcebook. London: Sage, 2014

Noblit, George; Hare, Dwight R. Meta-Ethnography: Synthesizing Qualitative Studies. London: Sage, 1988

Olarte-Mejía, D. V.; Ríos-Osorio, L. "Enfoques y estrategias de responsabilidad social implementadas en Instituciones de Educación Superior. Una revisión sistemática de la literatura científica de los últimos 10 años". Revista de la Educación Superior. Julio-Septiembre, 2015, pp. 19-40. [http:// www.scielo.org.mx/pdf/resu/v44n175/v44n175a2.pdf]

Oliver, Paul. Writing your Thesis. London: Sage, 2014.

Onwuegbuzie, A.; Frels, R. 7 Steps to a Comprehensive Literature Review: A Multimodel \& Cultural Approach. Sage: London, 2016.

Pan, M. L. Prepating Literature Reviews. Qualitative and Quantitative Approaches. London: Routlegde, 2017.

Petticrew, Mark; Roberts, Helen. Systematic Reviews in the Social Sciences. Malden: Blackwell, 2006.

Ridley, Diana. The Literature Review. London: Sage, 2012.

Rodríguez, Maria Luisa; Llanes, Juan (coords.). Cómo Elaborar, Tutorizar y Evaluar un Trabajo de Fin de Máster. Barcelona: Agència per a la Qualitat del Sistema Universitari de Catalunya, 2013

Sandelowski, Margarete; Barroso, Julie. Handbook for Synthesizing Qualitative Research. New York: Springer, 2007.

Urquhart, C. "Systematic reviewing, meta-analysis and meta-synthesis for evidence-based library and information science" Information Research, 2010, 15(3). [http://InformationR.net/ir/15-3/colis7/colis708.html]

Vállez, Mari; Codina, Lluís (2018). "Periodismo comput cional: evolución, casos y herramientas". El profesional de la información, v. 27, n. 4, pp. 759-768. https://doi. org/10.3145/epi.2018.jul.05

Yin, Robert K. Qualitative Research from Start to Finish. New Uork: Guilford Press, 2010.

Yin, Robert K. Case Study Research: Design and Methods. London: Sage, 2014.

Nota: este y los dos capítulos precedentes, son una versión actualizada del Framework ReSiste CSH publicado previamente en abril 2018 (ver referencia Codina, L. 2018). 


ISBN 978-84-09-20524-0

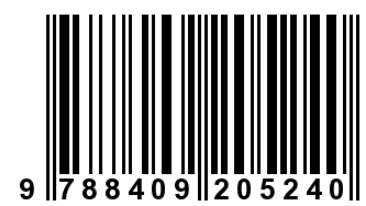

Máster Universitario en Investigación en Comunicación Social (MUCS)

\begin{tabular}{l|ll}
$\mathbf{u} p f$. & $\begin{array}{l}\text { Universitat } \\
\text { Pompeu Fabra } \\
\text { Barcelona }\end{array}$ & $\begin{array}{l}\text { DIGIDOC Grup de Recerca } \\
\text { en Documentació Digital } \\
\text { i Comunicació Interactiva }\end{array}$
\end{tabular} 